\title{
Mortalidad de vertebrados silvestres en la carretera Troncal del Caribe, Magdalena, Colombia
}

\section{Mortality of vertebrate wildlife in the highway Troncal del Caribe, Magdalena, Colombia}

\author{
María A. Adárraga-Caballero y Luis C. Gutiérrez-Moreno
}

\section{Resumen}

Las redes viales causan grandes impactos sobre el medio ambiente, desde la mortalidad directa de animales silvestres por atropello, hasta la reducción en la conectividad del paisaje y el aislamiento de poblaciones naturales. En Colombia, los estudios sobre atropello de fauna son incipientes y aún existen muchos vacíos en la generación de información primaria, especialmente en las carreteras de la costa Caribe. Por ello se seleccionaron para este estudio dos segmentos de carreteras en la región: 1) la vía Parque Nacional Natural Isla Salamanca (45 km de longitud) y 2) la vía del Parque Nacional Natural Tayrona ( $34 \mathrm{~km})$. En ambas se realizaron recorridos mensuales a bordo de un vehículo a una velocidad máxima de $30 \mathrm{~km} / \mathrm{h}$, durante 5 meses, comprendidos entre octubre de 2016 y enero de 2017. Se registraron un total de 208 atropellos (46 especies identificadas), y se encontró que los reptiles y mamíferos son los grupos mayormente afectados en los segmentos 1 y 2 , respectivamente. Por último, se calculó la tasa de atropello (TA) para cada especie por carretera; destacándose las especies Boa constrictor y Procyon cancrivorus, para las cuales se hallaron una de las TA más altas reportadas en Latinoamérica.

Palabras clave. Atropello. Caribe colombiano. Carreteras. Índice de atropello. Vertebrados.

\begin{abstract}
Road networks cause great impacts on the environment, from the direct mortality of wild animals that are hit by moving vehicles to the reduction of connectivity in the landscape and the isolation of natural populations. In Colombia, studies on wildlife roadkill are incipient, and there are still many gaps in the generation of primary information, especially on the roads of the Caribbean coast. For this reason, we selected two road stretches in the Colombian Caribbean region: 1) Isla Salamanca National Natural Park highway (45 km) and 2) Tayrona National Natural Park highway $(34 \mathrm{~km})$. On both roads, monthly trips were made on board a vehicle at a maximum speed of $30 \mathrm{~km} / \mathrm{h}$ during 5 months, between October 2016 and January 2017. We recorded a total of 208 roadkills (46 identified species), being reptiles and mammals the most affected groups in segments 1 and 2, respectively. Finally, we calculated the roadkill rate (TA) for each species. The species Boa constrictor and Procyon cancrivorus stand out because they obtained highest TA values reported in Latin America.
\end{abstract}

Keywords. Colombian Caribbean. Highways. Roadkill. Roadkill rate. Vertebrates. 


\section{Introducción}

Los proyectos viales son generalmente percibidos como obras que impulsan el desarrollo social y económico de las regiones, pues ofrecen múltiples beneficios a las poblaciones humanas (Arroyave et al., 2006; Peñaloza et al., 2011). En las últimas décadas, América Latina ha experimentado un fuerte crecimiento poblacional, asociado a un proceso de urbanización intenso y descontrolado (Sosa et al., 2011). Esto ha desencadenado un acelerado desarrollo de las redes viales, que son los principales canales de transporte de pasajeros y cargas. En Colombia, por ejemplo, los proyectos viales de cuarta generación $(4 \mathrm{G})$, planean la intervención de más de $8000 \mathrm{~km}$ de carreteras nacionales, $141 \mathrm{~km}$ en túneles, $150 \mathrm{~km}$ de viaductos y aproximadamente $1370 \mathrm{~km}$ de nuevas dobles-calzadas (Andrade, 2014).

Sin embargo, a pesar de los múltiples y evidentes beneficios a las poblaciones humanas, las redes viales (carreteras, caminos y trochas) constituyen una de las perturbaciones antropogénicas con mayores impactos al medio ambiente (Renjifo, 1999; Vargas, 2011). Los efectos directos implican la mortalidad de fauna silvestre por colisión vehicular (Fahrig et al., 1995; Gibbs \& Shriver, 2002; Garrah et al., 2015), mientras que los efectos indirectos están relacionados con la reducción en la conectividad poblacional y de paisaje, derivando en múltiples problemas a nivel de especies, poblaciones y comunidades (Forman \& Alexander, 1998; Seiler, 2001; Rojas, 2010; Vargas, 2011; Torres, 2011; Castillo et al., 2015; Garrah et al., 2015). En las últimas tres décadas, el rápido avance en proyectos viales ha causado que las cifras de fauna muerta por atropellos aumenten drásticamente (Forman \& Alexander, 1998; Rojas, 2010). Esta situación deriva en un peligroso desequilibrio en las poblaciones naturales, especialmente de aquellas especies que se encuentran bajo algún grado de amenaza (Monroy et al., 2015) o que sufren la perdida sistemática de individuos por esta causa.

Estudios desarrollados principalmente en Australia, Europa y Norteamérica (Dodd et al., 2003; van der Ree et al., 2011) concluyen que las pérdidas de fauna por colisión vehicular ameritan más investigación para la toma de medidas eficaces a la hora de la construcción de nuevas redes viales y en el manejo de las ya existentes (Fahrig \& Rytwinski, 2009; Rojas, 2010).

Por otra parte, América Latina es una de las regiones del mundo con mayor perspectiva de expansión vial. Sin embargo, los estudios en ecología de carreteras aún son escasos (González \& Benítez, 2013) y en algunos países las medidas preventivas apenas están tomando forma. Los países con más estudios producidos alrededor de la ecología de carreteras en el Neotrópico son Brasil, México y Costa Rica, y en su mayoría las investigaciones apuntan a la identificación de la fauna atropellada en carreteras nacionales y los impactos que estas generan sobre algunas especies y poblaciones biológicas particulares. Sin embargo, estudios más recientes se han enfocado en la determinación de hotspots como mecanismo para la identificación de puntos de cruce de fauna silvestre y su utilización en la elección acertada de medidas de mitigación (Tabeni et al., 2004; Teixeira et al., 2013; Artavia, 2015; Saranholi et al., 2016)

Aunque en Colombia la inversión en infraestructura vial ha ido en aumento, los estudios que dimensionan la problemática de los efectos de las carreteras sobre sistemas naturales protegidos o bosques circundantes son pocos. Algunos no han sido publicados y otros son solo reportes periodísticos (Monroy et al., 2015). Aunque algunos autores han afirmado que el país se encuentra aún en "estado embrionario" (Payán et al., 2013), recientemente se han hecho esfuerzos por producir datos alrededor de esta problemática en varias zonas del territorio nacional, destacándose estudios como los desarrollados en Antioquia (Delgado, 2007), Valle del Cauca (Vargas et al., 2011), Quindío (Quintero-Ángel et al., 2012; López et al., 2016), Guajira (Corpoguajira \& Fundación Biota, 2012), Valle del Magdalena (Payán et al., 2013), Popayán (Castillo et al., 2015), y Santander (Ramos \& Meza Joya, 2018), entre otros, y concluyendo siempre la urgente necesidad de llenar los vacíos existentes a nivel nacional en materia de ecología de carreteras.

Para la costa Caribe colombiana, la Ruta Nacional 90 (también llamada Transversal o Troncal del Caribe), es el corredor vial más importante para la conectivi- 
dad de la región con el resto del país. Esta vía atraviesa, bordea y constituye la vía de acceso a dos áreas protegidas importantes en la región: el complejo Vía Parque Isla Salamanca y el Parque Nacional Natural Tayrona en el departamento del Magdalena (tramos siete y ocho, respectivamente), cuya fauna asociada se ve obligada a salvar esta barrera para acceder a los recursos que se distribuyen a lado y lado de la carretera. Según observaciones particulares, actualmente los mecanismos para la mitigación de muertes de fauna silvestre en estas dos carreteras son insuficientes o inexistentes; por ejemplo, en el caso de la carretera asociada a Vía Parque Isla Salamanca, solo existen algunas señales de precaución para el paso de fauna, mientras que en la vía que conduce al Parque Nacional Natural Tayrona, estos mecanismos están completamente ausentes, probablemente debido a la falta de conocimiento de la problemática y por lo tanto a la aparente ausencia del problema.
Es por ello que el presente estudio se plantea como objetivo generar datos acerca de las cifras de mortalidad de fauna silvestre, a través de la estimación de la tasa de atropellos de las especies afectadas en los dos segmentos de carretera seleccionados.

\section{Materiales y métodos}

Área de estudio. La carretera Transversal del Caribe (Ruta Nacional 90), es el corredor vial más importante del Caribe colombiano y uno de los tres más relevantes a nivel nacional. Está asociada a dos áreas de interés para la conservación de la biodiversidad: el complejo Vía Parque Isla Salamanca (segmento 1) y el Parque Nacional Natural Tayrona (segmento 2). Ambos segmentos constituyen el área de estudio del presente trabajo (Figura 1).

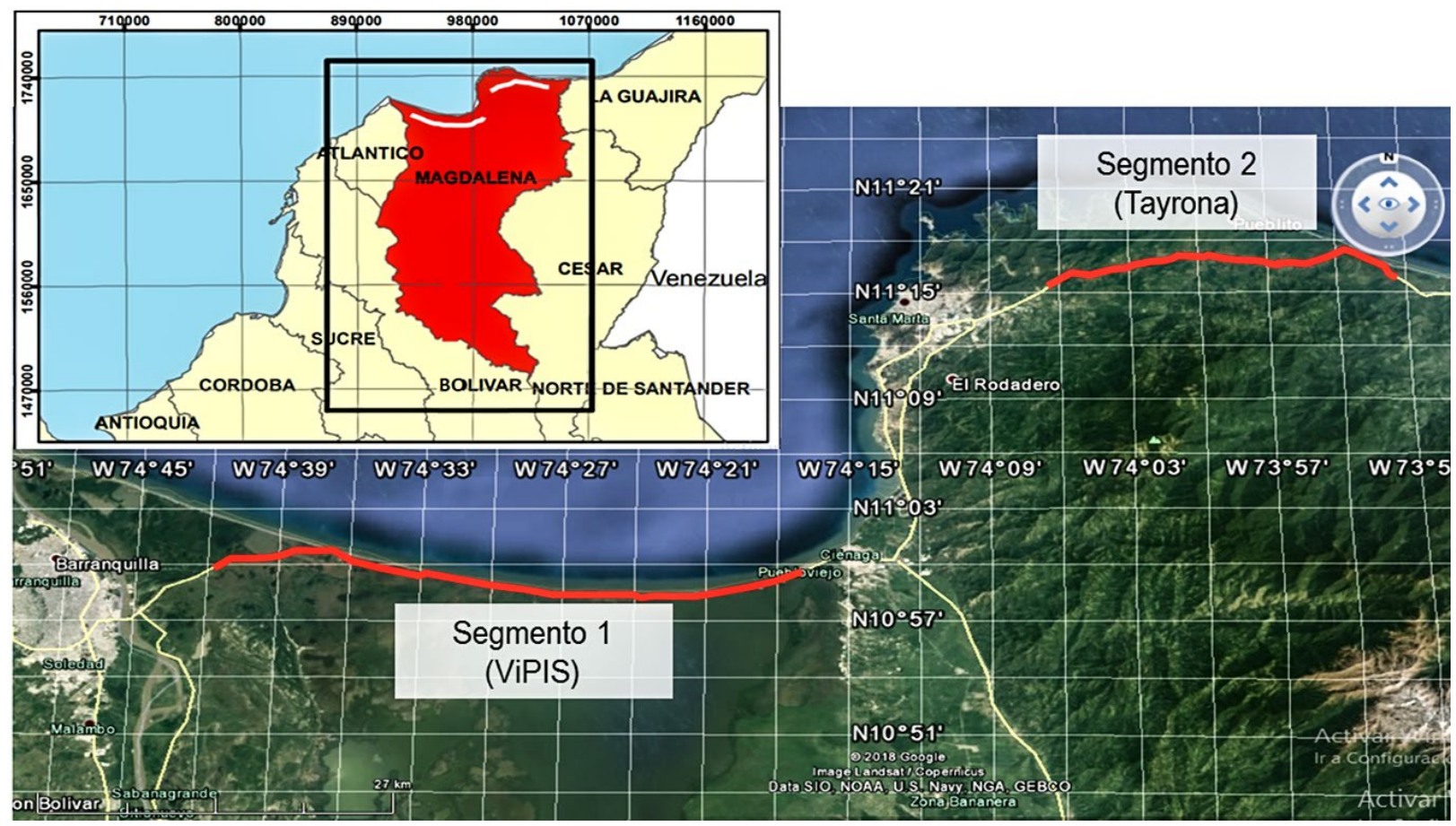

Figura 1. Segmentos 1 (ViPIS) y 2 (Tayrona) sobre la de la carretera troncal del Caribe, Magdalena, Colombia. Tomado y modificado de https://www.google.com.co/maps. 
Segmento 1 (S-1, ViPIS); con punto de inicio en $10^{\circ} 58^{\prime} 43.38^{\prime \prime} \mathrm{N}-74^{\circ} 43^{\prime} 46.89^{\prime \prime} \mathrm{O}$ y punto de finalización en $10^{\circ} 58^{\prime} 37.08^{\prime \prime} \mathrm{N}-74^{\circ} 20^{\prime} 9.21^{\prime \prime} \mathrm{O}$ : vía asfaltada que discurre entre la ciudad de Barranquilla en el departamento del Atlántico y la localidad de Ciénaga, en el departamento del Magdalena (Figura 1). Con una longitud de $45.27 \mathrm{~km}$, atraviesa por el costado occidental la reserva Nacional Natural Vía Parque Isla Salamanca, ubicado en la provincia biogeográfica Cinturón Árido Pericaribeño, dentro del distrito biográfico delta del Magdalena (Latorre, 2005). El parque es una importante reserva de ecosistemas de características estuarinas, con aproximadamente 12,000 hectáreas de bosques de manglar (Sesana, 2006) que se ven fragmentadas en dos sectores bien diferenciados (norte y sur) por el paso de la carretera; también cuenta con bosques inundables, pantanos costeros, arbustales, pastos, canales, ciénagas y playas, entre otros. La carretera es, en su mayoría, recta, con algunas curvas moderadas en algunos sectores y algunas señales de tránsito que indican el paso de fauna silvestre.

Segmento 2 (S-2, Tayrona); con punto de inicio en $11^{\circ} 15^{\prime} 11.43^{\prime \prime} \mathrm{N}-74^{\circ} 6^{\prime} 33.19^{\prime \prime} \mathrm{O}$ y punto de finalización en $11^{\circ} 14^{\prime} 54.71^{\prime \prime} \mathrm{N}-73^{\circ} 50^{\prime} 22.46^{\prime \prime} \mathrm{O}$ : vía asfaltada que discurre entre el corregimiento de Bonda y el Río Guachaca, ambos en jurisdicción del departamento del Magdalena (Figura 1). Con una longitud aproximada de $35 \mathrm{~km}$, bordea el área de influencia del Parque Nacional Natural Tayrona, el cual alberga unidades vegetales como el matorral espinoso, el bosque subxerofítico (Barbosa et al., 2012) y uno de los relictos de bosque seco tropical mejor conservados de Colombia (Pizano \& García, 2014). La carretera posee tramos rectos, con algunas curvas moderadas y otras cerradas en algunos sectores. No existe señalización que indique el paso de fauna.

Colección de datos. Durante 5 meses continuos, comprendidos entre octubre de 2016 y febrero de 2017, se realizaron 18 recorridos a lo largo de cada uno de los segmentos, para un total de 36 muestreos y 1432.98 kilómetros de carretera. Debido a diversos motivos como disponibilidad del vehículo o de los observadores, el número de recorridos mensuales varió entre tres y cuatro, y se cubrieron tanto el periodo lluvioso (oc- tubre-noviembre) como el periodo seco (diciembre-febrero), propios del régimen climático bimodal descrito para para la zona (Sánchez et al., 2006). Los recorridos fueron diurnos (entre 6:00 y las 9:00 horas, aproximadamente), lo que permitió reportar aquellos animales que habían sido atropellados durante la noche (Carvajal \& Díaz, 2013; Artavia, 2015). Fueron efectuados por dos observadores y un conductor a bordo de un vehículo, a una velocidad que osciló entre 20 y $30 \mathrm{~km} / \mathrm{h}$. Aunque algunas investigaciones con métodos similares se han ejecutado a velocidades mayores (Artavia, 2015), el estudio realizado por Collinson et al. (2014) señala que para este tipo de trabajos, los $50 \mathrm{~km} / \mathrm{h}$ es la velocidad limite antes que la detección de los atropellamientos empiece a ser inexacta.

Se registraron los cadáveres de animales atropellados correspondientes a fauna silvestre (no se contabilizaron especímenes domésticos). De cada registro se tomaron datos referentes a la posición geográfica con un GPS Garmin eTrex 30. La identificación taxonómica se realizó in situ; cuando los cadáveres estaban en mal estado, se identificó hasta la categoría más baja posible (Arroyave et al., 2006; Rojas, 2010).

Análisis de datos. Para la detección de grupos y especies más y menos afectados se hizo uso de análisis de estadística descriptiva, incluyendo análisis de frecuencias y agrupación de datos. Después de verificar la normalidad de los datos por medio de la prueba de Shapiro-Wilk, se aplicó una prueba de Análisis de Varianza (ANOVA) para determinar la existencia de diferencias significativas entre los atropellos registrados en ambos periodos climáticos, por medio del uso del paquete estadístico de uso gratuito Past v 3.16.

A partir del registro de los atropellos y su distribución a lo largo de los meses de muestreo se calculó la tasa de atropello (TA) (Seijas et al., 2013; Payan et al., 2013)que corresponde a una medida de frecuencia que relaciona el número de atropellos con el número de kilómetros recorridos en un tiempo determinado.

Tasa de atropellos $(\mathrm{TA})=\mathrm{N}$ atropellos $/(\mathrm{N} \mathrm{km}$ ${ }^{*} \mathrm{~N}$ recorridos) 
Este índice se obtuvo de manera individual para cada una de las especies afectadas en ambos segmentos estudiados, tomando en cuenta los kilómetros totales recorridos a lo largo de los 5 meses de muestreo.

Estos análisis se llevaron a cabo mediante el paquete estadístico SPSS v 15.0 (SPSS Inc 2006).

\section{Resultados}

Se registraron un total de 208 eventos de atropello, 131 para el segmento 1 (ViPIS) y 77 para el segmento 2 (Tayrona), distribuidos por clase taxonómica como lo muestra la Tabla 1 . Se identificaron un total de 46 especies afectadas, 32 en el segmento 1 y 25 en el segmento 2 (Tabla 1 ).

Tabla 1. Distribución de los registros de atropello de fauna en la troncal del Caribe, Magdalena, Colombia.

\begin{tabular}{|c|c|c|c|c|c|c|c|c|c|}
\hline \multirow{3}{*}{ GRUPO } & \multicolumn{6}{|c|}{ SEGMENTOS } & \multirow{2}{*}{\multicolumn{3}{|c|}{ Total (S1+S2) }} \\
\hline & \multicolumn{3}{|c|}{ S1 (ViPIS) } & \multicolumn{3}{|c|}{ S2 (Tayrona) } & & & \\
\hline & Spp & $\mathbf{N}$ & $\%$ & Spp & $\mathbf{N}$ & $\%$ & Spp & $\mathbf{N}$ & $\%$ \\
\hline Anfibios & 2 & 4 & 3.05 & 2 & 16 & 20.78 & 3 & 20 & 9.62 \\
\hline Reptiles & 9 & 48 & 36.64 & 9 & 15 & 19.48 & 13 & 63 & 30.3 \\
\hline Aves & 15 & 39 & 29.77 & 6 & 9 & 11.69 & 21 & 48 & 23.1 \\
\hline Mamíferos & 6 & 40 & 30.53 & 8 & 37 & 48.05 & 9 & 77 & 37 \\
\hline TOTAL & 32 & 131 & 62.98 & 25 & 77 & 37.01 & 46 & 208 & 100 \\
\hline
\end{tabular}

Los mayores índices de atropello se presentaron durante el periodo lluvioso en ambos segmentos; así mismo, el menor valor para el segmento 1 se encuentra asociado también a este periodo climático, mientras que en el segmento 2 se encuentra relacionado al periodo seco.

A pesar de ello la prueba de varianza (ANOVA), evidenció que no existían diferencias estadística- mente significativas entre el número de atropellos reportados en cada uno de los periodos climáticos (seco y lluvioso) para cada uno de los segmentos estudiados (Segmento 1= 0.9192; Segmento 2=0.2509), por lo cual se entiende que para el presente estudio las lluvias no son un factor determinante en la distribución en el tiempo de la abundancia de los atropellos. 
A nivel taxonómico, se presentan diferencias en las tasas de atropellamiento (TA) generales entre los segmentos. Los grupos con mayor y menor frecuencia variaron en cada uno de los segmentos estudiados. Por ejemplo, para el segmento 1 (Tabla 1), los reptiles y los anfibios fueron los grupos de mayor y menor frecuencia respectivamente (Figura 2), mientras que para el segmento 2, estos lugares son ocupados por los mamíferos y las aves (Tabla 1 ).

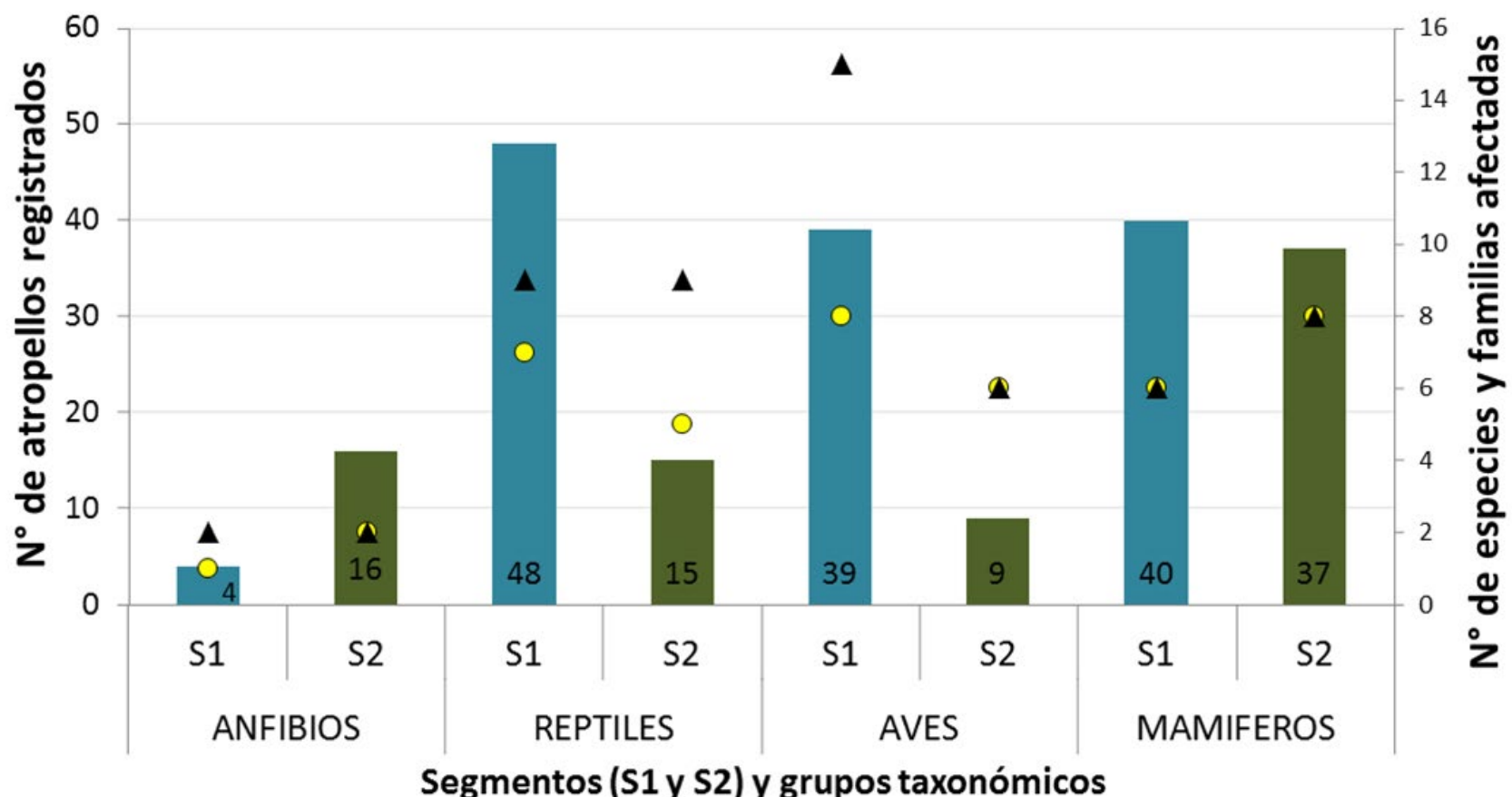

$\mathrm{N}^{\circ}$ Registros S1 y S2 O Familias $\Delta$ Especies

Figura 2. Frecuencia de grupos taxonómicos afectados por colisión vehicular en dos segmentos de la Troncal del Caribe, Magdalena, Colombia.

La riqueza especifica de fauna atropellada también fue variable entre grupos y entre segmentos (Figura 2 ), siendo las aves en el segmento 1 el grupo de mayor riqueza, con 15 especies afectadas, entre las que se contaron especies migratorias como Protonotaria citrea. Por su parte, los anfibios resultaron ser el grupo con menor número de especies involucradas en accidentes para ambos segmentos, con solo tres taxones reportados (Figura 2).
El índice de atropellamiento calculado para el Segmento 1 es de 0.16 Ind./ km, y para el segmento 2 es de 0.12 Ind./ km (Tabla 2).

Las tasas de atropello calculadas para cada una de las especies identificadas se reportan en la Tabla 2. Se destacan particularmente los altos valores registrados para especies como Boa constrictor y Procyon cancrivorus, con 0.022 y 0.021 Ind. $/ \mathrm{km}$, respectivamente. 
Tabla 2. Tasas de atropello (TA) calculadas para cada una de las especies identificadas en la troncal del Caribe, Magdalena, Colombia. NI: Registros sin identificación taxonómica, *Especies con las tasas de atropellos (TA) más altas calculadas en este estudio.

\begin{tabular}{|c|c|c|c|c|c|c|c|c|}
\hline \multirow{4}{*}{ Orden } & \multirow{4}{*}{ Familia } & \multirow{4}{*}{ Especie } & \multirow{2}{*}{\multicolumn{2}{|c|}{$\frac{\text { S1 }}{\text { (ViPIS) }}$}} & \multicolumn{2}{|c|}{ S2 } & \multirow{2}{*}{\multicolumn{2}{|c|}{$\frac{\text { Total }}{(\mathrm{S} 1+\mathrm{S} 2)}$}} \\
\hline & & & & & & rona) & & \\
\hline & & & \multicolumn{2}{|c|}{$814.86 \mathrm{~km}$} & \multicolumn{2}{|c|}{$618.12 \mathrm{~km}$} & \multicolumn{2}{|c|}{$1432.98 \mathrm{~km}$} \\
\hline & & & $\mathbf{N}$ & TA & $\mathbf{N}$ & TA & $\mathbf{N}$ & TA \\
\hline Anura & Bufonidae & Rhinella marina & 2 & 0.0025 & 15 & 0.0243 & 17 & 0.012 \\
\hline Anura & Bufonidae & Rhinella granulosa & 1 & 0.0012 & - & - & 1 & 0.001 \\
\hline Anura & Leptodactylidae & Leptodactylus sp. & - & - & 1 & 0.0016 & 1 & 0.001 \\
\hline Anura & NI & Anuro NI & 1 & 0.0012 & - & - & 1 & 0.001 \\
\hline \multicolumn{2}{|c|}{ Subtotal anfibios (3 spp.) } & & 4 & & 16 & & 20 & \\
\hline Squamata & Dipsadidae & Thamnodynastes sp. & 1 & 0.0012 & - & - & 1 & 0.001 \\
\hline Squamata & Dipsadidae & Leptodeira annulata & - & - & 1 & 0.0016 & 1 & 0.001 \\
\hline Squamata & Dipsadidae & Pseudoboa neuwiedii & - & - & 1 & 0.0016 & 1 & 0.001 \\
\hline Squamata & Dipsadidae & Phimophis guianensis & 2 & 0.0025 & 1 & 0.0016 & 3 & 0.002 \\
\hline Squamata & Boidae & Boa constrictor* & 27 & 0.0331 & 4 & 0.0065 & 31 & $* 0.022$ \\
\hline Squamata & Boidae & Corallus ruschenbergerii & - & - & 2 & 0.0032 & 2 & 0.001 \\
\hline Squamata & Boidae & Epicrates maurus & 3 & 0.0037 & - & - & 3 & 0.002 \\
\hline Squamata & Boidae & Epicrates sp. & - & - & 1 & 0.0016 & 1 & 0.001 \\
\hline Squamata & Boidae & Serpiente NI & 1 & 0.0012 & - & - & 1 & 0.001 \\
\hline Squamata & Viperidae & Crotalus durissus & 1 & 0.0012 & 1 & 0.0016 & 2 & 0.001 \\
\hline Squamata & Colubridae & Mastigodryas sp. & 1 & 0.0012 & - & - & 1 & 0.001 \\
\hline Squamata & Colubridae & Spilotes pullatus & - & - & 1 & 0.0016 & 1 & 0.001 \\
\hline Squamata & Serpiente NI & Serpiente NI & 1 & 0.0012 & - & - & 1 & 0.001 \\
\hline Squamata & Iguanidae & Iguana iguana & 7 & 0.0086 & 3 & 0.0049 & 10 & 0.007 \\
\hline Squamata & Teiidae & Cnemidophorus lemniscatus & 3 & 0.0037 & - & - & 3 & 0.002 \\
\hline Crocodylia & Alligatoridae & Caiman crocodilus fuscus & 1 & 0.0012 & - & - & 1 & 0.001 \\
\hline \multicolumn{2}{|c|}{ Subtotal reptiles (13 spp.) } & & 48 & & 15 & & 63 & \\
\hline Anseriformes & Anatidae & Cairina moschata & - & - & 1 & 0.0016 & 1 & 0.001 \\
\hline Columbiformes & Columbidae & Columba livia & - & - & 1 & 0.0016 & 1 & 0.001 \\
\hline Cuculiformes & Cuculidae & Crotophaga ani & 1 & 0.0012 & - & - & 1 & 0.001 \\
\hline Cuculiformes & Cuculidae & Coccyzus americanus & 2 & 0.0025 & - & - & 2 & 0.001 \\
\hline Pelecaniformes & Ardeidae & Butorides virescens & 2 & 0.0025 & - & - & 2 & 0.001 \\
\hline Pelecaniformes & Ardeidae & Bubulcus ibis & 2 & 0.0025 & - & - & 2 & 0.001 \\
\hline Pelecaniformes & Ardeidae & Ardea alba & 2 & 0.0025 & - & - & 2 & 0.001 \\
\hline Pelecaniformes & Ardeidae & Ardea sp. & 3 & 0.0037 & - & - & 3 & 0.002 \\
\hline Pelecaniformes & Ardeidae & Egretta tricolor & 1 & 0.0012 & - & - & 1 & 0.001 \\
\hline Cathartiformes & Cathartidae & Cathartes aura & 3 & 0.0037 & - & - & 3 & 0.002 \\
\hline Accipitriformes & Accipitridae & Accipiter bicolor & 1 & 0.0012 & - & - & 1 & 0.001 \\
\hline Accipitriformes & Accipitridae & Buteogallus meridionalis & 1 & 0.0012 & - & - & 1 & 0.001 \\
\hline Accipitriformes & Accipitridae & Rupornis magnirostris & 1 & 0.0012 & - & - & 1 & 0.001 \\
\hline
\end{tabular}




\begin{tabular}{|c|c|c|c|c|c|c|c|c|}
\hline Coraciiformes & Alcedinidae & Megaceryle alcyon & 1 & 0.0012 & - & - & 1 & 0.001 \\
\hline Piciformes & Ramphastidae & Pteroglossus torquatus & - & - & 1 & 0.0016 & 1 & 0.001 \\
\hline Psittaciformes & Psittacidae & Eupsittula pertinax & - & - & 1 & 0.0016 & 1 & 0.001 \\
\hline Passeriformes & Tyrannidae & Empidonax sp. & - & - & 1 & 0.0016 & 1 & 0.001 \\
\hline Passeriformes & Tyrannidae & Tiránido NI & - & - & 1 & 0.0016 & 1 & 0.001 \\
\hline Passeriformes & Troglodytidae & Troglodytes aedon & 2 & 0.0025 & - & - & 2 & 0.001 \\
\hline Passeriformes & Icteridae & Molothrus sp. & 1 & 0.0012 & - & - & 1 & 0.001 \\
\hline Passeriformes & Parulidae & Protonotaria citrea & 12 & 0.0147 & - & - & 12 & 0.008 \\
\hline Passeriformes & Cardinalidae & Saltator coerulescens & - & - & 1 & 0.0016 & 1 & 0.001 \\
\hline Ave NI & Ave NI & Ave NI & 4 & 0.0049 & 2 & 0.0032 & 6 & 0.004 \\
\hline \multicolumn{2}{|c|}{ Subtotal aves (21 spp.) } & & 39 & & 9 & & 48 & \\
\hline Didelphimorphia & Didelphidae & Didelphis marsupialis & 3 & 0.0037 & 22 & 0.0356 & 25 & 0.017 \\
\hline Pilosa & Myrmecophagidae & Tamandua mexicana & 2 & 0.0025 & 1 & 0.0016 & 3 & 0.002 \\
\hline Chiroptera & Noctilionidae & Noctilio sp. & 1 & 0.0012 & - & - & 1 & 0.001 \\
\hline Chiroptera & Murciélago NI & Murciélago NI & 1 & 0.0012 & - & - & 1 & 0.001 \\
\hline Carnivora & Canidae & Cerdocyon thous & - & - & 6 & 0.0097 & 6 & 0.004 \\
\hline Carnivora & Felidae & Leopardus sp. & 1 & 0.0012 & 1 & 0.0016 & 2 & 0.001 \\
\hline Carnivora & Mephitidae & Conepatus semistriatus & - & - & 2 & 0.0032 & 2 & 0.001 \\
\hline Carnivora & Procyonidae & Procyon cancrivorus ${ }^{*}$ & 29 & 0.0356 & 1 & 0.0016 & 30 & ${ }^{*} 0.021$ \\
\hline Rodentia & Muridae & Rattus rattus & 1 & 0.0012 & 1 & 0.0016 & 2 & 0.001 \\
\hline Rodentia & Roedor NI & Roedor NI & 2 & 0.0025 & - & - & 2 & 0.001 \\
\hline Rodentia & Sciuridae & Sciurus granatensis & - & - & 1 & 0.0016 & 1 & 0.001 \\
\hline Mamífero NI & Mamífero NI & Mamífero NI & - & - & 2 & 0.0032 & 2 & 0.001 \\
\hline \multicolumn{2}{|c|}{ Subtotal mamíferos (9 spp.) } & & 40 & & 37 & & 77 & \\
\hline Total (46 spp.) & & & 131 & 0.1608 & 77 & 0.1246 & 208 & 0.145 \\
\hline
\end{tabular}

Es de destacar que el $77 \%$ de los registros de reptiles para el segmento 1 son ofidios. La especie Boa constrictor (boa común) presentó atropellos en todos los meses de muestreo, para un total de 31 registros en ambas carreteras y una tasa de atropello general (TA) de 0.021 Ind./ $\mathrm{km}$, siendo más alto para el segmento 1 , donde se registraron la mayor cantidad de especímenes impactados.

\section{Discusión}

La tasa de atropello (TA) para el segmento 1 (0.16 Ind./ $\mathrm{km}$ ) fue ligeramente más alta que la calculada para el segmento 2 (0.12 Ind./ $\mathrm{km}$ ). Al ser comparadas con las obtenidas en otros estudios en el Neotrópico, se encuentra que se aproximan a las halladas en investigaciones como la llevada a cabo por Seijas et al. (2013) en Venezuela (TA: 0.22 Ind./ km), pero son menores a las encontradas por algunos otros autores como Coelho et al., (2008) en Brasil (TA: 0.34), Monroy et al. (2015) (TA: 0.32 Ind. $/ \mathrm{km}$ ) y De La Ossa-V. \& Galván-Guevara (2015) (TA 0.32 Ind./ km) en Colombia.

Cabe resaltar que las diferencias en metodologías, características geográficas y ecológicas de las áreas de estudio y tipo de carreteras consideradas, hacen difícil la comparación de las tasas de atropellos entre distintas investigaciones realizadas en el Neotrópico, incluso de aquellas realizadas en el mismo país (Ferreira da Cunha et al., 2010).

Para el presente estudio los grupos más y menos afectados variaron en cada segmento. Los grupos con mayor número de atropellos para esta investigación, coinciden con lo hallado en algunas investigaciones desarrolladas en el Neotrópico en donde resultan ser los reptiles (Morales et al., 1997; Seijas et al., 2013; De La Ossa-Nad- 
jar \& De La Ossa-V., 2015) y los mamíferos (Pinowski, 2005; Coelho et al., 2008; Ferreira da Cunha et al., 2010; Castillo et al., 2015; De La Ossa-V. \& Galván-Guevara, 2015) los grupos más afectados.

Boa constrictor (boa común), la especie con la frecuencia de atropello más alta, con 31 registros totales (29 en segmento 1 y 4 en segmento 2), obtuvo el mayor índice de atropello calculado para una especie en el presente trabajo (0.021 Ind./ km), especialmente en el segmento 1 , donde alcanzó un valor de $0.33 \mathrm{Ind} . / \mathrm{km}$. Por su parte, para Procyon cancrivorus (mapache cangrejero), se obtuvo un índice de atropello similar, tanto para los registros totales $(0.020 \mathrm{Ind} . / \mathrm{km})$ como para el segmento 1 (0.036 Ind./ $\mathrm{km}$ ), en donde se registraron la mayor cantidad de individuos, superando ligeramente a B. constrictor.

Aunque algunos autores reportan cantidades de registros similares (Tabla 3), los índices de atropello (Ind./ km) calculados para B. constrictor y P. cancrivorus en investigaciones anteriores, son menores a los hallados en el presente estudio (Tabla 3), situándolo, hasta el momento, como uno de los índices de atropello más altos calculado para ambas especies a nivel del Neotrópico.

Tabla 3. Comparativo de los índices de atropello para Boa constrictor y Procyon cancrivorus hallados en estudios para el Neotrópico. *Valores calculados para el presente estudio a partir de los datos aportados por cada autor, excepto Seijas et al. (2013) y Payán et al. (2013) quienes aportan el índice de atropello para cada especie de manera individual.

\begin{tabular}{|c|c|c|c|c|c|c|}
\hline \multicolumn{3}{|c|}{ Identificación del estudio } & \multicolumn{2}{|c|}{ B. constrictor } & \multicolumn{2}{|c|}{ P. cancrivorus } \\
\hline Autores & País & km & Ind. & Ind./km* & Ind. & Ind./km* \\
\hline Coelho et al. (2008) & Brasil & $195^{*}$ & -- & -- & 21 & SD \\
\hline Cáceres et al. (2010) & Brasil & 3900 & -- & -- & 15 & 0.0038 \\
\hline Ferreira da Cunha et al. (2010) & Brasil & 21600 & 4 & 0.0001 & 7 & 0.0003 \\
\hline Omena et al. (2012) & Brasil & 11877 & 7 & 0.0005 & -- & -- \\
\hline Seijas et al. (2013) & Venezuela & 2080 & 5 & 0.0026 & 9 & 0.0043 \\
\hline Payán et al. (2013) & Colombia & 2753.4 & 19 & 0.0069 & 4 & 0.0015 \\
\hline De La Ossa-V. \& Galván-Guevara (2015) & Colombia & 1305.6 & 6 & 0.0045 & 8 & 0.0061 \\
\hline Monroy et al. (2015) & Colombia & 2352 & 5 & 0.0021 & -- & -- \\
\hline Artavia (2015) & Costa Rica & 3036.8 & 30 & 0.0098 & -- & -- \\
\hline Saranholi et al. (2016) & Brasil & 7200 & 1 & 0.0001 & 1 & 0.0001 \\
\hline Este estudio (2019) & Colombia & 1432.9 & 31 & 0.0216 & 30 & 0.0209 \\
\hline
\end{tabular}

A pesar de que las tasas reportadas para ambas especies son comparativamente altas, no necesariamente significan un impacto negativo significativo a nivel poblacional (Fahrig \& Rytwinski, 2009); si bien los atropellos generan pérdidas importantes a nivel de las poblaciones, es necesario evaluar el estado de las mismas a nivel regional para determinar en qué medida estos índices de atropello las afectan (López et al., 2016).

La alta abundancia de reptiles para el segmento 1 puede estar asociada a su necesidad de termorregulación, siendo la carretera un factor atrayente debido al calor 
que el asfalto puede transferirles (López et al., 2016), tanto en el día como en la noche (Arroyave et al., 2006). Esto ha sido señalado en numerosas investigaciones como una característica altamente influyente en la tasa de atropellos, especialmente en el caso de las serpientes (Ashley \& Robinson, 1996), coincidiendo con lo reportado para el segmento 1 , donde la mayoría de los registros de reptiles $(77.08 \%)$ corresponden a ofidios, como lo reportado por Castillo et al. (2015) y López et al. (2016). Adicionalmente, se ha establecido que individuos de algunas especies de serpientes se inmovilizan ante la aproximación de un vehículo, asumiendo una postura estática o defensiva (Shine et al., 2004; Andrews \& Whitfield, 2005; Castillo et al., 2015; López et al., 2016), lo que podría estar contribuyendo al alto del índice de atropellos para este grupo.

Para el segmento 2, el grupo con la mayor cantidad de registros fueron los mamíferos, especialmente representados por un alto número de muertes para la especie Didelphis marsupialis (zarigüeya), la cual compone el $59.45 \%$ de los registros de mamíferos para esta vía. La alta frecuencia de atropellos de esta especie coincide con lo reportado en otros estudios para el Neotrópico (Delgado, 2007; Payán et al., 2013; Seijas et al., 2013; Artavia, 2015; Castillo et al., 2015; De La Ossa-V. \& Galván-Guevara, 2015), y puede estar relacionada a sus reconocidos hábitos omnívoros y oportunistas, aprovechando los cadáveres de otros atropellos como fuente de alimento. Pinowski (2005) señala que D. marsupialis es una especie de atropello frecuente, que puede encontrarse asociada tanto a hábitats naturales como a antropogénicos.

Las muertes de D. marsupialis ocurren mayoritariamente en la noche o de madrugada. Esta especie es comúnmente percibida como una especie nociva, debido a que se le asocia con la depredación de aves de corral y frutos cultivados (Aponte, 2013), por lo que algunos conductores los atropellan de manera intencional; esto, sumado a su andar torpe sobre tierra (Arcangeli, 2014), la poca movilidad para evadir los vehículos y su comportamiento de inmovilización al ser deslumbrados por las luces de los mismos (Omena et al., 2012), son factores que pueden influir sobre los altos índices de muerte de esta especie en carretera. Lo anterior también se puede aplicar a Cerdocyon thous (zorro perro), que ocupa el segundo lugar en cuanto a mamíferos atropellados para el segmento 2, y que ha sido señalada como una de las especies con mayor número de muertes por colisión vehicular en algunas zonas del Neotrópico (Pinowski, 2005; Delgado, 2007).

Las aves ocuparon el tercer lugar en número de registros. Sin embargo, presentan la riqueza más alta, con 21 especies reportadas a nivel general (Tabla 2), siendo mucho más abundantes en el segmento 1, que en el segmento 2 ( 39 y 9 registros, respectivamente). Esta diferencia en las abundancias puede estar vinculada a las características de la vegetación que se dispone en el borde de cada uno de los segmentos y a la biología del grupo en sí. Debido a su capacidad de vuelo, buena parte de las aves tienden a desplazarse en un rango de altura similar a la de la vegetación que está dispuesta en los bordes de la carretera. Adicionalmente, la diferencia en la oferta de recursos en los ecosistemas alrededor de ambas carreteras puede estar influyendo en las tasas de accidentalidad para el grupo, de manera que en lugares con mayor oferta de alimento o refugio, la necesidad de movilizarse sea menor. En general, las aves resultan ser uno de los grupos taxonómicos menos afectados en cuanto a número de atropellos (Seijas et al., 2013; De la Ossa-Nadjar \& De La Ossa-V., 2015; Monroy et al., 2015), lo cual probablemente esté relacionado, entre otros aspectos, al vuelo, que les otorga una ventaja sobre los otros grupos para evadir los vehículos con más eficiencia.

La afectación de especies de aves migratorias como Empidonax sp., Coccyzus americanus, Megaceryle alcyon y especialmente Protonotaria citrea (que presentó uno de los índices de atropello más altos para una especie de ave) (Tabla 2) se debe principalmente a la coincidencia de los meses de muestreo con las épocas de migración (Naranjo et al., 2012), tiempo durante el cual son especialmente vulnerables (Gómez \& Najera, 2000) debido a sus desplazamientos frecuentes.

Finalmente, la ausencia de diferencias significativas entre los atropellos en el periodo seco y lluvioso para 
ambos segmentos indica que los regímenes de lluvias no influyen significativamente en la distribución de los atropellos durante el presente estudio, lo cual coincide con lo reportado por Torres (2011) en un estudio similar desarrollado en Costa Rica.

\section{Conclusiones}

El tramo 7 de la carretera Troncal del Caribe (segmento 1) produce actualmente las tasas de atropello más altas reportadas para Latinoamérica de las especies Procyon cancrivorus y Boa constrictor. Se recomienda llevar a cabo estudios poblacionales de ambas especies, con el fin de determinar si la muerte sistemática de individuos incide significativamente en la salud de las poblaciones a nivel local y regional.

Los grupos taxonómicos que resultan mayor y menormente afectados en las carreteras estudiadas varían dependiendo de varios factores propios de las vías y del paisaje al cual se asocian.

La posible afectación deliberada de especies que se consideran peligrosas o dañinas (Langley et al., 1989; Quintero-Ángel et al., 2012), puede contribuir a los altos índices de atropello de serpientes como la boa (Boa constrictor) y mamíferos como zorros (Cerdocyon thous) y zarigüeyas (Didelphis marsupialis), por lo cual es urgente generar medidas que promuevan la educación de los usuarios de las vías en torno al papel de estas especies en sus ecosistemas.

En cuanto al grupo taxonómico de las aves, es probable que durante las épocas de migración, las especies de aves migratorias de alta movilidad como la reinita cabecidorada (Protonotaria citrea), se vean más afectadas que las especies residentes, por lo cual sería interesante la implementación de medidas estacionales para la reducción de la velocidad durante las temporadas migratorias.

Es probable que factores como la altura de la vegetación en los bordes de las vías incida sobre las tasas de atropello de grupos de alta movilidad como las aves, ya que estas tienden a desplazarse entre las líneas de vegetación que se encuentra a ambos lados de la carretera, por lo que a mayor altura de la cobertura vegetal menor riesgo de colisión. Se recomienda que los próximos estudios de ecología de carreteras en el país incluyan la búsqueda de relaciones entre factores del paisaje y los atropellos de fauna, ya que el conocimiento de estas puede contribuir a la canalización de medidas de mitigación efectivas que vayan más allá de la colocación de señales de paso de fauna, que generalmente son ignoradas por los conductores.

\section{Referencias}

Andrade, L. F. (2014). Encadenamientos e infraestructura. (Informe técnico). Bogotá D.C., Colombia: Agencia Nacional de Infraestructura (ANI). 22 pp.

Andrews, K. \& Whitfield, J. (2005). How do highways influence snake movement? Behavioral responses to roads and vehicles. Copeia, 4, 772-782.

Aponte, J. D. (2013). Una revisión de la biología del Didelphis marsupialis y su relación con el mal de Chagas y la leishmaniasis. Hipótesis, Apuntes Científicos Uniandinos, Número Especial, 96-101.

Arcangeli, J. (2014). Manejo de crías de zarigüeya (Didelphis virginiana) en cautiverio. Revista Electrónica de Veterinaria REDVET, 15(9), 1-13.

Arroyave, M., Gómez, C., Guitiérrez, M., Múnera, D., Zapata, P., Vergara, I. Andrade L., \& Ramo, K. (2006). Impactos de las carreteras sobre la fauna silvestre y sus principales medidas de manejo. EIA (Escuela de ingenieria de Antioquia), 5, 45-57.

Artavia, R. (2015). Identificación y caracterización de cruces de fauna silvestre en la sección de la ampliación de la carretera nacional Ruta 32, Limón, Costa Rica. (Tesis de grado). Turrialba, Costa Rica: CATIE.

Ashley, P. \& Robinson, J. T. (1996). Road mortality of amphibians, reptiles and other wildlife on the Long Point Causeway, Lake Erie, Ontario. Canadian Field Naturalist, 110(3).

Barbosa, R., Castro, A., Cifuentes, Y., Díaz, J., Gómez, F., Ramírez, V., Yela, W., Castro, S., González, S., Cortes, D., Currea, S., Florez, G., Calderón, L., Calderón, M., Duque ,F., González, L., Henao, M., Landínez, M., 
\&Guzmán, V. (2012). Estudio regional continental del PNN Tayrona y zonas aledañas (Rodadero). Bogotá.

Cáceres, N., Hannibal, W., Freitas, D., Silva, E., Roman, C. \& Casella, J. (2010). Mammal occurrence and roadkill in two adjacent ecoregions (Atlantic Forest and Cerrado) in south-western Brazil. Zoologia (Curitiba), 27(5), 709-117.

Carvajal, V. \& Díaz, F. (2013). Atropello de mamíferos silvestres en la ruta de acceso al cantón de Liberia, Guanacaste, Costa Rica. Ventana, 7(1), 12-14.

Castillo, J. C., Urmendez, D. \& Zambrano, G. (2015). Mortalidad de fauna por atropello Vehicular en un sector de la Vía Panamericana entre Popayán y Patía. Boletín científico Centro de Museos Museo de Historoa Natural, 19(2462-8190), 207-219.

Coelho, I. P., Kindel, A. \& Coelho, A. V. P. (2008). Roadkills of vertebrate species on two highways through the Atlantic Forest Biosphere Reserve, southern Brazil. European Journal of Wildlife Research, 54(4), 689-699.

Collinson, W. J., Parker, D. M., Bernard, R. T. F., Reilly, B. K. \& Davies-Mostert, H. T. (2014). Wildlife road traffic accidents: a standardized protocol for counting flattened fauna. Ecology and Evolution, 4(15), 3060-71.

Corpoguajira \& Fundacion Biota. (2012). Caracterización y monitoreo de la mortalidad de pequeños mamíferos silvestres en los corredores viales del departamento de la Guajira. Riohacha, Colombia: Corpoguajira y Fundación Biota.

De La Ossa-Nadjar, O. \& De La Ossa V., J. (2015). Vehicle collisions with wild fauna on the two roads that pass through the Montes De María, Sucre, Colombia. Revista U.D.C.A Actualidad \& Divulgación Científica, 18(2), 503-511.

De La Ossa-V., J. \& Galván-Guevara, S. (2015). Registro de mortalidad de fauna silvestre por colisión vehicular en la carretera Toluviejo-ciénaga La Caimanera, Sucre, Colombia. Biota Colombiana, 16(1), 67-77.

Delgado-V., C. A. (2007). Muerte de mamíferos por vehículos en la Vía del Escobero, Envigado (Antioquia), Colombia. Actualidades Biológicas, 29(87), 229-233.

Dodd, C. K., Barichivich, W. J. \& Smith, L. L. (2003). Effectiveness of a barrier wall and culverts in reducing wildlife mortality on a heavily traveled highway in Florida. Biological Conservation, 118(5), 619-631.

Fahrig, L., Pedlar, J. H., Pope, S. E., Taylor, P. D. \& Wegner, J. F. (1995). Effect of Road Traffic on Amphibian Density. Biological Conservation, 73, 177-182.
Fahrig, L. \& Rytwinski, T. (2009). Effects of roads on animal abundance: an empirical review and synthesis. Ecology and Society, 14(1), 21.

Ferreira da Cunha, H.., Moreira, F. G. A. \& Silva, S. D. S. (2010). Roadkill of wild vertebrates along the GO-060 road between Goiânia and Iporá, Goiás State, Brazil. Acta Scientiarum. Biological Sciences, 32(3), 257-263.

Forman, R. T. T. \& Alexander, L. E. (1998). Roads and their major ecological effects. Annual Review of Ecology and Systematics, 29(1), 207-231.

Garrah, E., Danby, R. K., Eberhardt, E., Cunnington, G. M. \& Mitchell, S. (2015). Hot spots and hot times: Wildlife road mortality in a regional conservation corridor. Environmental Management, 56(4), 874-889.

Gibbs, J. \& Shriver, W. (2002). Estimating the effects of road mortality on turtle populations. Conservation Biology, 16(6), 1647-1652.

Gómez, P. \& Najera, J. (2000). Fauna silvestre víctima de las carreteras: el caso de Costa Rica. Repertorio Científico, 6, 47-50.

González, A. G. \& Benítez, G. (2013). Road ecology studies for Mexico: A review. Oecologia Australis, 17(171), 175-190.

Langley, W. M., Lipps, H. W. \& Theis, J. F. (1989). Responses of Kansas motorists to snake models on a rural highway. Transactions of the Kansas Academy of Science (1903), 92(1/2), 43.

Latorre, J. P. (2005). Biodiversidad y conservación en los Parques Nacionales Naturales de Colombia, Una Aproximación Histórico-Geográfica a Escala 1:1.000.000.

López, D. F., León-Yusti, M., Guevara-Molina, S. C. \& Vargas-Salinas, F. (2016). Reptiles en corredores biológicos y mortalidad por atropellamiento vehicular en Barbas-Bremen, departamento del Quindío, Colombia. Revista de La Academia Colombiana Ciencias Exactas Fisicas y Naturales, 40(156), 484-493.

Monroy, M. C., De La Ossa-Lacayo, A., \& De La Ossa-V, J. (2015). Tasa de atropellamiento de fauna silvestre en la Vía San Onofre-María La Baja, Caribe colombiano. Asociación Colombiana de Ciencias Biológicas, 1(27), 88-95.

Morales Mávil, J. E., Villa-Cañedo, J. T., Aguilar Rodríguez, S. H. \& Barragán Morales, L. (1997). Mortalidad de vertebrados silvestres en una carretera asfaltada de la región de Los Tuxtlas, Veracruz, México. La Ciencia y El Hombre, 27, 7-23. 
Naranjo, L. G., Amaya, J. D., Eusse-González, D. \& Cifuentes-Sarmiento, Y. (2012). Guía de las especies migratorias de la biodiversidad en Colombia. Aves. Bogotá D. C., Colombia: Ministerio de Ambiente y Desarrollo Sostenible, WWF Colombia.

Omena Junior, R., Pantoja Lima, J., Wendt Santos, A. L., Aguiar ribeiro, G. A. \& Rocha Aride, P. H. (2012). Caracterizacion de fauna vertebrada atropellada en la via BR-174; Amazonas Brasil. Revista Colombiana de Ciencia Animal, 4(2), 291-307.

Payán, E., Soto, C., Díaz-Pulido, A., Coordinador, L., Benítez, A. \& Hernández, A. (2013). Wildlife Road Crossing And Mortality: Lessons For Wildlife Friendly Road Design In Colombia. International Conference on Ecology and Transportation at Scottsdale.Volume: 209D, 1-4.

Peñaloza, C., Mojica, A., Muñoz, M., Escobar, M., Torrado, C., Silva, L., Perez, G. \& Ramos, L. (2011). Diagnóstico del transporte, 2011. Bogotá D. C.: Ministerio Nacional de Transporte de Colombia, 112 pp.

Pinowski, J. (2005). Roadkills of vertebrates in Venezuela. Revista Brasileira de Zoologia, 22(1), 191-196.

Pizano, C. \& García, H. (Eds.). (2014). El Bosque seco Tropical en Colombia . Bogotá D.C., Colombia: Instituto de Investigacion de Recursos Biológicos Alevander von Humboldt.

Quintero-Ángel, A., Osorio-Domínguez, D., Vargas-Salinas, F. \& Saavedra-Rodríguez, C. A. (2012). Roadkill rate of snakes in a disturbed landscape of Central Andes of Colombia. Herpetology Notes, 5, 99-105.

Ramos, E. \& Meza Joya, F. L. (2018). Reptile road mortality in a fragmented landscape of the middle Magdalena Valley, Colombia. Herpetology Notes, 11, 81-91.

Renjifo, L. M. (1999). Composition changes in a subandean avifauna after long-term forest fragmentation. Conservation Biology, 13(5), 1124-1139.

Rojas, E. (2010). Atropello de vertebrados en una carretera secundaria en Costa Rica. Research Journal of the Costa Rican Distance Education University, 3(1), 81-84.

Sánchez, G., Hernández ,M., Mayor, G., Gómez, C., Corredor, I., Puentes, M., Blanco, W., Muñoz, M., Pinzón, J., Franke, R. \& Equipo de Operarios del Parque. (2006). Plan de manejo Parque Nacional Natural Tayrona, Unidad de Parques. Santa Marta: Direccion Territorial Caribe. 298 pp.
Saranholi, B. H., Bergel, M. M., Ruffino, P. H., Rodríguez-C, K. G., Ramazzotto, L. A., de Freitas, P. D. \& Galetti M, P. (2016). Roadkill hotspots in a protected area of Cerrado in Brazil: planning actions to conservation. Revista MVZ Córdoba, 21(2), 5441-5448.

Seijas, A. E., Araujo-Quintero, A. \& Velásquez, N. (2013). Mortalidad de vertebrados en la carretera Guanare-Guanarito, estado Portuguesa, Venezuela. Revista de Biologia Tropical, 61(4), 1619-1636.

Seiler, A. (2001). Ecological effects of roads, A review. Grimsö Wildlife Research Station, Dept. of Conservation Biology, University of Agricultural Sciences, 9, 40.

Sesana, L. (2006). Colombia Natural Parks. Bogotá, D. C.: Villegas S.A.

Shine, R., Lemaster, M., Wall, M., Langkilde, T. \& Mason, R. (2004). Why did the snake cross the road? Effects of roads on movement and location of mates by garter snakes (Thamnophis sirtalis parietalis). Ecology and Society, 9(1), 1-9.

Sosa, A. J., Kogan, J. H., Azán, S., Miquilena, M. E. \& Alcántara de Vasconcellos, E. (2011). Desarrollo urbano y movilidad en América Latina. Banco de desarrollo de America Latina CAF. Banco de Desarrollo de América Latina.

Tabeni, M. S., Bender, J. B., \& Ojeda, R. A. (2004). Puntos calientes para la conservación de mamíferos en la provincia de Tucumán, Argentina. Mastozoología Neotropical, 11(1), 55-67.

Teixeira, F. Z., Coelho, I. P., Esperandio, I. B., Oliveira, N. R., Peter, F. P., Dornelles, S. S., Delazeri, N. R., Tavares, M., Martins, M. B. \& Kindel, A. (2013). Are road-kill hotspots coincident among different vertebrate groups? Oecologia Australis, 17(1), 36-47.

Torres, M. L. (2011). Funcionalidad de estructuras subterráneas como pasos de fauna en la carretera Interamericana Norte que cruza el Área de Conservación Guanacaste, Costa Rica. Costa Rica: Catie. 134 pp.

Van Der Ree, R., Jochen A. G., J., Van Der Grift, E. A. \& Clevenger, A. P. (2011). Effects of roads and traffic on wildlife populations and landscape function : road ecology is moving toward larger scales. Ecology and Society, 16(1), 9 .

Vargas, F. S., Delgado Ospina, I. \& López Aranda, F. (2011). Mortalidad por atropello vehicular y distribución de anfibios y reptiles en un bosque subandino en el occidente de Colombia. Caldasia, 33(1), 121-138. 
María A. Adárraga-Caballero

Universidad del Atlántico

Barranquilla, Colombia

madarraga@gmail.com

https://orcid.org/0000-0003-3384-0500

Luis C. Gutiérrez-Moreno

Universidad del Atlántico

Barranquilla, Colombia

rotifero15@gmail.com

https://orcid.org/0000-0002-0353-381X
Mortalidad de vertebrados silvestres en la carretera Troncal del Caribe, Magdalena, Colombia

Citación del artículo: Adárraga-Caballero, M. A. \& Gutiérrez-Moreno, L. C. (2019). Mortalidad de vertebrados silvestres en la carretera Troncal del Caribe, Magdalena, Colombia. Biota Colombiana, 20(1), 106-119. DOI: 10.21068/c2019.v20n01a07.

Recibido: 21 de febrero de 2018

Aceptado: 29 de abril de 2019 